\title{
3GPP Spectrum Access Evolution Towards 5G
}

\author{
Michal Szydelko ${ }^{1, *}$ and Marcin Dryjanski ${ }^{2}$ \\ ${ }^{1}$ Movilo Ltd., Milostowska 8/9, 51-315 Wroclaw, Poland \\ ${ }^{2}$ Grandmetric Ltd., Malta Office Park, Baraniaka 88E/F, 61-131 Poznan, Poland
}

\section{Abstract}

The ever-increasing needs for more spectrum resources, and the emerging new Radio Access Technologies under the 5G umbrella add to the complexity of the Spectrum Toolbox in mobile networks landscape. This article covers $3 \mathrm{GPP}$ LTE evolution from Release 8 up to the Release 14, which deals with the LTE-Advanced Pro enhancements. A collection of available frequency bands, spectrum aggregation mechanisms, licensing and duplexing schemes, as well as spectrum sharing and refarming techniques is described. With such a classification, Spectrum Toolbox is defined and its evolution directions are discussed, with the opportunities and challenges of the individual features summarized. Studies on the new non-backwards compatible Radio Access Technology, as well as the new channel models for higher frequency bands are also covered. The presented Spectrum Toolbox is considered as a baseline for the introduction of the new air interface framework towards $5 \mathrm{G}$ ecosystem in the context of future mobile networks enhancements.

Keywords: 3GPP evolution, 5G, LTE-A Pro, spectrum management, Spectrum Toolbox, WRC-15.

Received on 15 May 2016, accepted on 20 August 2016, published on 23 February 2017

Copyright (C) 2017 M. Szydelko and M. Dryjanski, licensed to EAI. This is an open access article distributed under the terms of the Creative Commons Attribution licence (http://creativecommons.org/licenses/by/3.0/), which permits unlimited use, distribution and reproduction in any medium so long as the original work is properly cited.

doi: 10.4108/eai.23-2-2017.152184

\section{Introduction}

The ever-increasing mobile data demand in the cellular networks is expected to cover growing variety of applications ranging from Mobile Broadband (MBB) to the Internet-of-Things (IoT) and vehicular communications. This calls for more spectrum resources and for novel spectrum access schemes providing higher flexibility of the radio resources allocation. This in turn will increase the overall complexity of the radio resources management (RRM) in the mobile networks. Recent 3GPP Rel-13/14 standardization within the Radio Access Networks (RAN) group, and discussions during 3GPP workshops on "5G RAN" and on the Licensed-Assisted Access (LAA), gave clear indication of the raising requirements for further spectrum allocation flexibility improvements.

Different spectrum bands ranging from $450 \mathrm{MHz}$ up to $100 \mathrm{GHz}$, covering licensed, unlicensed and licensed-shared allocations, together with various spectrum access methods

*Corresponding author. Email: michal.szydelko@movilo.net and optimization techniques create a complex spectrum landscape.

To address and classify various aspects of such a landscape, this article presents the Spectrum Toolbox, which serves as a guide through spectrum-related solutions that are already available or being currently studied within global standardization bodies. The Spectrum Toolbox analysis covers:

- Frequency bands overview,

- Spectrum aggregation mechanisms,

- Spectrum licensing,

- Duplexing schemes,

- Spectrum sharing,

- Spectrum refarming techniques.

Based on the 3GPP RAN standardization status ${ }^{\dagger}$, a brief introduction to the available solutions is presented, focusing on LTE and WLAN radio technologies and related spectrum

${ }^{\dagger}$ As of $2016 / 04$ 
aspects with an outlook on further 3GPP standards development towards $5 \mathrm{G}$.

The remainder of this article is organized as follows: after a brief introduction on selected radio access standardization and regulatory events, section 3 outlines an overview of the 3GPP solutions for spectrum access techniques, classifying Spectrum Toolbox elements. In section 4, a discussion on the potential future standardization development directions of the Spectrum Toolbox is presented, followed by the final conclusions in section 5 .

\section{Setting up the scene for Spectrum Toolbox discussion}

This chapter presents outcomes of recent radio access standardization and regulatory events, including LAA workshop, "5G RAN" workshop, and World Radio Conference (WRC-15). These discussions set up the scene for the evaluation of Spectrum Toolbox, covered in the following sections.

\subsection{GPP LAA workshop}

3GPP RAN workshop on the Licensed-Assisted Access collected inputs from various unlicensed spectrum stakeholders, including IEEE802 committee, Wi-Fi Alliance (WFA), Wireless Broadband Alliance (WBA), and from the regulatory bodies' representatives [1]. The goal of the workshop was to strengthen the technical collaboration, especially in the areas of coexistence evaluation for the unlicensed Industrial, Scientific and Medical (ISM) bands among different actors and to follow up with the finalization of the LAA feature in the 3GPP specifications. The main conclusions of the workshop were related to the Listen Before Talk (LBT) mechanism, which provides fair coexistence between LTE and WLAN users within $5 \mathrm{GHz}$ ISM band. Coexistence testing and performance requirements definitions discussion was also started, focusing on DL-only operation of LAA scheme within 3GPP Rel-13 timeframe".

\subsection{G RAN-related workshops}

3GPP workshop on "5G RAN" development directions collected ideas and requirements on the next generation mobile networks, including 5G timeline feasibility discussion [2]. In terms of spectrum allocations for $5 \mathrm{G}$, below $6 \mathrm{GHz}$ spectrum and millimeter wave $(\mathrm{mmW})$ spectrum bands were discussed for new, non-backward compatible 5G Radio Access Technology (RAT) denoted as NR (i.e. New Radio of $5 \mathrm{G}$ ). NR will be developed in parallel to the evolution of LTE-Advanced $\mathrm{Pro}^{\S}$ in the coming 3GPP releases. As a facilitator for the technical feasibility studies and performance benchmarks, work on the mmW channel models will have to be concluded first in Rel-14 study item [3], covering spectrum bands ranging from $6 \mathrm{GHz}$ to up to $100 \mathrm{GHz}^{* *}$.

During early 2016, another 3GPP RAN workshop on channel model for frequency in the range of $6-100 \mathrm{GHz}$ was held. Number of technical agreements related to the channel modeling was reached, related to such aspects as:

- Channel model calibration methods,

- Line-of-Sight (LOS) probability,

- UE rotation motion,

- Building penetration loss,

- Atmospheric loss,

- Foliage loss,

- Ground reflection,

- Dynamic blockage aspects, and many more.

Additionally, the 3GPP has recognized channel modeling work performed in the other standardization bodies, as well as in the related European "Horizon 2020" research projects like METIS, MiWEBA, and mmMagic ${ }^{\dagger \dagger}$.

Table 1. WRC-15 decisions on spectrum allocation for 4G mobile services [4]

\begin{tabular}{|c|c|c|}
\hline Frequency bands & Geographical distribution & Spectrum availability \\
\hline $470-694 / 698 \mathrm{MHz}$ & Some APAC and American countries & Auction in the USA \\
\hline 694-790 MHz & Global band, now including EMEA & $60 \mathrm{MHz}$ \\
\hline 1427-1518 MHz & Global band, in most countries & $91 \mathrm{MHz}$ \\
\hline $3300-3400 \mathrm{MHz}$ & Global band, not Europe/North America & $100 \mathrm{MHz}$ \\
\hline $3400-3600 \mathrm{MHz}$ & Global band, in most countries & $200 \mathrm{MHz}$ \\
\hline $3600-3700 \mathrm{MHz}$ & Global band, not Africa/some APAC & $100 \mathrm{MHz}$ \\
\hline $4800-4990 \mathrm{MHz}$ & Some APAC and American countries & $190 \mathrm{MHz}$ \\
\hline \multicolumn{3}{|c|}{$\begin{array}{l}\text { \$ UL LAA to be covered in Enhanced LAA (eLAA) Rel-14 work item. } \\
\S \text { LTE-Advanced Pro defined as LTE evolution in Rel-13 and beyond. } \\
* \text { 5G RAT will be defined for both below and above } 6 \mathrm{GHz} \text { spectrum. } \\
{ }^{*} \text { Projects details: https://5g-ppp.eu/5g-ppp-phase-1-projects/ }\end{array}$} \\
\hline
\end{tabular}




\subsection{WRC-15 conference}

WRC-15 conference aimed to allocate new frequency bands for mobile services, ranging from road safety to global flight tracking use cases [4]. New spectrum allocation was agreed for MBB communication services in L-band (i.e. 1427$1518 \mathrm{MHz}$ ), and in C-band (i.e. $3.4-3.6 \mathrm{GHz}$ ). More detailed WRC-15 spectrum allocation is presented in Table 1 .

During this conference, a decision on the spectrum agenda studies for the next WRC-19 conference was also taken, aiming at identification of the $5 \mathrm{G}$ frequency bands above $6 \mathrm{GHz}$. In these studies, the following spectrum bands are to be taken into consideration:
$24.25-27.5 \mathrm{GHz}$,
$31.8-33.4 \mathrm{GHz}$
$37-43.5 \mathrm{GHz}$, $45.5-50.2 \mathrm{GHz}, 50.4-52.6 \mathrm{GHz}, 66-76 \mathrm{GHz}, 81-86 \mathrm{GHz}$

Furthermore, it was decided that the broadcasting and mobile industry players in Europe have to conclude on the opportunity of MBB technologies adaptation for future terrestrial TV requirements, while using TV UHF band (i.e. $470-694 \mathrm{MHz})$. The consensus on this issue has to be reached until WRC-23 conference.

\section{Spectrum Toolbox}

The increasing amount of the available spectrum resources, equipped with a number of the spectrum access technologies, provides a complicated system to operate with a complex coordination of the resources among RAN nodes. Figure 1 presents an example of the Heterogeneous Network (HetNet) comprising of macro- and Small Cell (SC) layers, accompanied with various spectrum access techniques, including Carrier Aggregation (CA) and Dual Connectivity (DC). It utilizes both, licensed and unlicensed spectrum.

Spectrum Toolbox covers the available frequency bands, spectrum aggregation mechanisms, licensing and duplexing schemes, and spectrum sharing and refarming techniques. Table 2 presents the detailed overview of the Spectrum Toolbox evolution over the LTE releases, while the following sections elaborate on the individual areas presented therein.

Table 2. Spectrum Toolbox evolution across LTE releases: existing solutions, ongoing Study Items, and future concepts for $5 \mathrm{G}$ discussions

\begin{tabular}{|c|c|c|c|c|}
\hline 3GPP release & $\begin{array}{l}\text { LTE: } \\
\text { Rel-8, } 9\end{array}$ & $\begin{array}{c}\text { LTE-A: } \\
\text { Rel-10, 11, } 12 \\
\end{array}$ & $\begin{array}{l}\text { LTE-A Pro: } \\
\text { Rel-13, } 14\end{array}$ & $\begin{array}{l}5 G \text { phase I: Rel-15 } \\
5 G \text { phase II: Rel-16 }\end{array}$ \\
\hline $\begin{array}{c}\text { Frequency bands } \\
{[\mathrm{GHz}]^{\star}}\end{array}$ & $\begin{array}{c}0.7,0.8,1.8,2.1,2.3- \\
2.4 \\
2.5-2.6 \mathrm{GHz}\end{array}$ & $\begin{array}{c}0.45 \text { (Brazil), } \\
\text { Digital Dividend, 1.5, } \\
\text { 3.4-3.8GHz }\end{array}$ & $\begin{array}{c}5 \mathrm{GHz} \text { ISM; } \\
\text { WRC-15 bands }\end{array}$ & $\begin{array}{l}\text { New bands below } \\
6 \mathrm{GHz} \text { for } 5 \mathrm{G} \text { RAT; } \\
\text { mmW: } 6-100 \mathrm{GHz} ; \\
\text { WRC-15/19 bands }\end{array}$ \\
\hline $\begin{array}{l}\text { Spectrum } \\
\text { aggregation }\end{array}$ & $\begin{array}{c}\text { Single Carrier } \\
(1.4-20 \mathrm{MHz}), \\
\text { symmetric DL/UL }\end{array}$ & $\begin{array}{l}\text { Dual Connectivity, } \\
\text { CA variants: } \\
\text {-up to 5CC, } \\
\text {-intra-/ inter-band, } \\
\text {-(non)-continuous, } \\
\text {-FDD and/or TDD } \\
\text {-Co-located, RRH; } \\
\text {-asymmetric DL/UL }\end{array}$ & $\begin{array}{l}\text { Massive CA (up to } \\
\text { 32CC), } \\
\text { LAA (5GHz), LWA, } \\
\text { eLWA, SDL for CA: } \\
\text { 2.3-2.4GHz }\end{array}$ & $\begin{array}{l}\text { Multi-Connectivity with } \\
\text { asymmetric DL/UL, } \\
\text { SDL for CA: } \\
700 \mathrm{MHz} \text { and } \\
2.5-2.6 \mathrm{GHz}, \\
\text { Lean carrier }\end{array}$ \\
\hline $\begin{array}{l}\text { Spectrum licensing } \\
\text { schemes }\end{array}$ & $\begin{array}{l}\text { Licensed spectrum } \\
\text { only }\end{array}$ & $\begin{array}{l}\text { Licensed, } \\
\text { Carrier WiFi }\end{array}$ & $\begin{array}{c}\text { Licensed, } \\
\text { Unlicensed, } \\
\text { DL LAA, LWA, LSA, } \\
\text { eLWA }\end{array}$ & $\begin{array}{c}\text { Co-existence of: } \\
\text { exclusive licensed, } \\
\text { shared license-exempt } \\
\text { spectrum, enhanced } \\
\text { LAA (DL+UL) }\end{array}$ \\
\hline Duplexing schemes & Separate FDD, TDD & $\begin{array}{l}\text { FDD and TDD } \\
\text { (CA-based) } \\
\text { elMTA }\end{array}$ & FDD Flexible Duplex & $\begin{array}{c}\text { Full Duplex, } \\
\text { Additional DL-only TDD } \\
\text { configurations }\end{array}$ \\
\hline Sharing schemes ${ }^{* *}$ & $\begin{array}{c}\text { Static schemes } \\
(\mathrm{MOCN}, \mathrm{MORAN})\end{array}$ & $\begin{array}{c}\text { Static schemes } \\
\text { (MOCN, MORAN) }\end{array}$ & RSE, LSA & $\begin{array}{l}\text { LSA (new bands), } \\
\text { SC sharing, SCaaS, } \\
\text { spectrum trading, } \\
\text { Cognitive Radio }\end{array}$ \\
\hline Spectrum refarming & Static & Static & $\begin{array}{l}\text { Dynamic, DSA, } \\
\text { MRAT Joint } \\
\text { Coordination } \\
\end{array}$ & $\begin{array}{c}\text { Fully dynamic, } \\
\text { opportunistic, Cognitive } \\
\text { Radio }\end{array}$ \\
\hline
\end{tabular}




\subsection{Frequency bands}

List of the current $3 \mathrm{GPP}$ spectrum bands for E-UTRA is presented below [5]. Due to the complex nature of the country-, and market-specific spectrum bands allocation, the following compilation is limited only to presentation of the general spectrum bands without detailed geographical distinctions (all values are in $\mathrm{GHz}$ ):

- FDD: $0.45,0.7,0.8,0.9,1.5,1.8,1.9,2.1,2.3,2.6,3.5$

- TDD: $0.7,1.8,1.9,2.0,2.6,2.3-2.4,2.5-2.7,3.4-3.8$

Detailed E-UTRA spectrum bands arrangement is presented in the Figure 2, including different duplex modes, depicting Supplemental DL (SDL) carriers, and highlighting bands with the reversed DL/UL channels arrangement.

In the case of spectrum bands for the IEEE 802.11 technologies, unlicensed frequency bands are identified and summarized in Table 3.

As can be seen, LTE currently covers spectrum bands up to $4 \mathrm{GHz}$, while Wi-Fi is already standardized to operate within unlicensed part of the $\mathrm{mmW}$ spectrum, e.g. $60 \mathrm{GHz}$ band in case of WiGig standard. As already mentioned in the previous chapter, $5 \mathrm{G}$ is expected to bring the operation within much wider range of spectrum bands covering frequencies of up to $100 \mathrm{GHz}$ in both licensed and unlicensed variants.

\subsection{Channel bandwidths}

Baseline LTE standard (i.e. 3GPP Rel-8) was defined as an OFDMA $^{*}$ system supporting a single carrier with various predefined channel bandwidths (BW) in the range of $1.4-$ $20 \mathrm{MHz}$, i.e. $1.4,3,5,10,15,20$.
Recently, 3GPP Rel-14 study on the LTE bandwidth flexibility was agreed [6], with the aim to investigate further channel allocation flexibility enhancements to improve the spectrum utilization. This work is prompted by the fact, that in some countries, the channel arrangement plans results in a sub-optimal spectrum blocks allocations for mobile network operators, not corresponding to the nominal LTE Rel-8 channel BW variants. This can also happen in the case of spectrum refarming from GSM/UMTS to LTE (spectrum refarming is discussed in section 3.7). As claimed in [6], LTE non-compliant spectrum availability across regions is significantly varying resulting with the following spectrum blocks, e.g. 1.8, 2.0, 2.2, 4.4, 4.6, 6, 6.2, 7.8, 7.0, 8.0, 11, 14, $18,19[\mathrm{MHz}$. To address spectrum utilization in the LTE non-compliant spectrum blocks, it was proposed to study potential introduction of the generic radio framework. It should enable the system's operation in any spectrum blocks of sizes larger than $1.4 \mathrm{MHz}$ and smaller than $20 \mathrm{MHz}$. The described channel flexibility study refers to the previously considered concepts, such as New Carrier Type (NCT), or carrier segment with their respective RF impact analyses.

\subsection{Spectrum aggregation techniques}

In order to go beyond the spectrum availability offered by a single-carrier LTE as outlined in previous section, Rel-10 LTE-Advanced introduced CA feature to aggregate multiple Component Carriers (CC) with the use of MAC layer scheduling. Up to $5 \mathrm{CC}$ were allowed by Rel-10 where each

Figure 1. Spectrum toolbox landscape in HetNet

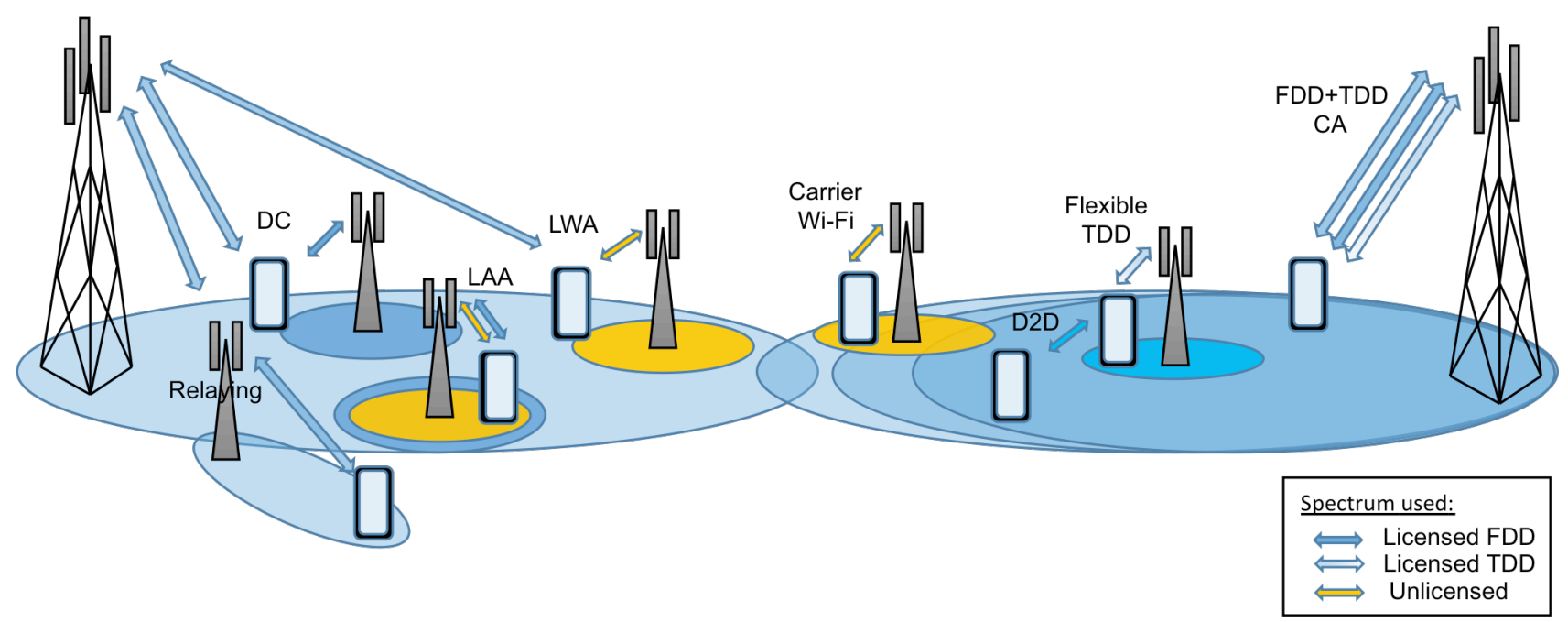

D2D - Device-to-Device

\footnotetext{
\# OFDMA used for LTE DL. For LTE UL, Single Carrier FDMA is used.
} 
Figure 2. E-UTRA frequency bands allocation chart

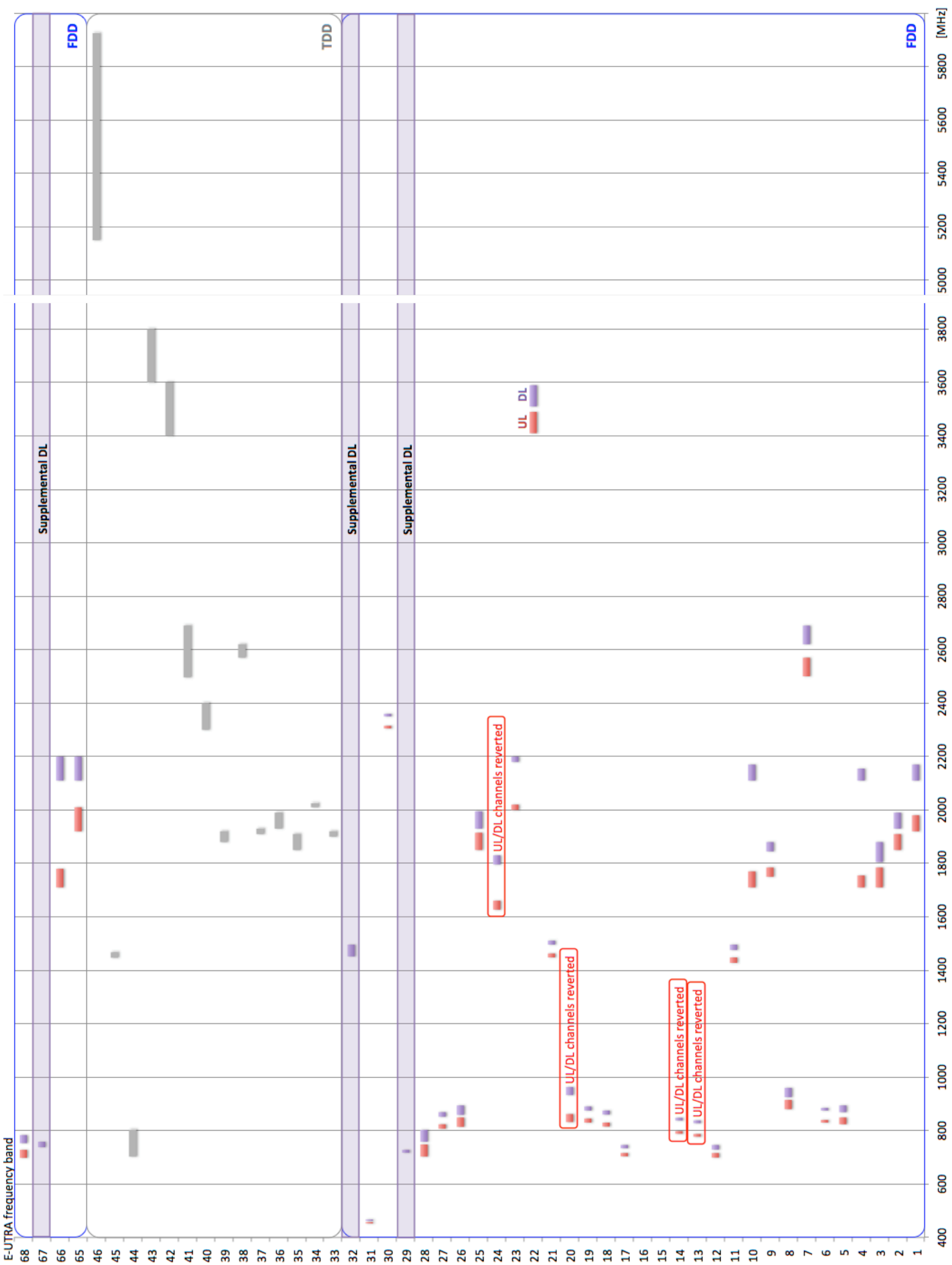


Table 3. IEEE802.11 spectrum bands breakdown for 802.11 protocol variants

\begin{tabular}{cccc}
\hline Protocol & Release date & Frequency band [GHz] & Channel bandwidth [MHz] \\
\hline 802.11 & 1997.06 & 2.4 & 22 \\
$802.11 \mathrm{a}$ & 1999.09 & $3.7^{*} ; 5$ & 20 \\
$802.11 \mathrm{~b}$ & 1999.09 & 2.4 & 22 \\
$802.11 \mathrm{~g}$ & 2003.06 & 2.4 & 20 \\
$802.11 \mathrm{n}$ & 2009.10 & $2.4 / 5$ & 20,40 \\
$802.11 \mathrm{ac}$ & 2013.12 & 5 & $20,40,80,160$ \\
$802.11 \mathrm{ad}$ & 2012.12 & 60 & 2160 \\
$802.11 \mathrm{ah}$ & $\sim 2016$ & 0.9 & $1,2,4,8,16$ \\
$802.11 \mathrm{aj}$ & $\sim 2016$ & $45 / 60$ & 540,1080 \\
802.11 ax & $\sim 2016$ & $2.4 / 5$ & 80,160 \\
802.11 ay & $\sim 2017$ & 60 & 8000 \\
\hline
\end{tabular}

* Licensed $3.7 \mathrm{GHz}$ band; allowed by FCC in the USA

individual $\mathrm{CC}$ is reusing the Rel-8 numerology for the $\mathrm{BW}$ size to allow backward compatibility. Thus, the overall theoretical maximum aggregated spectrum bandwidth summed up to $100 \mathrm{MHz}$ with intra-band consecutive, non-consecutive, or inter-band CA options. Different component carriers allocation for the UL and DL could reflect the expected traffic demand by the use of nonsymmetrical configurations (e.g. 3DL CC, 1UL CC). In addition, CA introduced concepts of the Primary Cell (PCell) and Secondary Cell (SCell), where the former is used for signaling and user data purposes, and the latter serves for the user data only to increase the overall user's throughput.

In terms of deployment scenarios, CA was standardized for either intra-site or inter-site ${ }^{\S \S}$ scenarios, based on the ideal backhaul due to the $\mathrm{CA}$ timing requirements on scheduling. In further releases, more flexibility in terms of the spectrum arrangement was allowed by joint aggregation of TDD- and FDD-based component carriers mixing.

Unpaired spectrum variant further improving the interband FDD CA operation was provided with the SDL concept, which allows addition of an unpaired ${ }^{* * *}$ DL carrier to the CA scheme. So far, there is limited number of SDL carriers standardized for the following bands:

- L-band SDL (i.e. 1452-1496 MHz, previously used for broadcasting services) as defined in [7],

- SDL for 717-728MHz band.

The discussion continues to introduce the harmonized European SDL band in the $738-758 \mathrm{MHz}$ range, and to enable SDL in the $2.6 \mathrm{GHz}$ band $[8,9]$. The already standardized SDL bands are depicted in Figure 2.

Even though 5CC Carrier Aggregation configuration was specified already in Rel-10, the highest CA configuration standardized for specific spectrum bands combination so far

\footnotetext{
$\S \S$ Based on RRH deployments using fibre for ideal backhaul.

*** Unpaired DL carrier - an FDD mode, where there is no accompanied/symmetric UL carrier. In this case, feedback in UL needs to be provided via the other UL carrier.
}

considers "only" up to 4 frequency bands for 4 component carriers [6], e.g.:

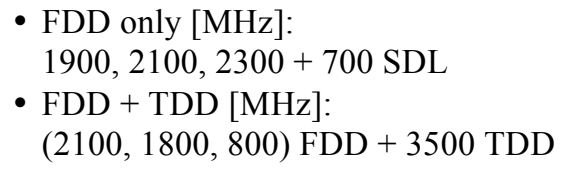

Lately, a work on the 5CC configuration for Carrier Aggregation has been started within Rel-13 for 5DL/1UL combination [10].

Significant change in the spectrum aggregation management was possible through the aggregation of different carriers with the use of Rel-12 Dual Connectivity (DC) feature. With DC, the spectrum is aggregated in intersite scenario, where a macro-cell serves as a mobility anchor (using the so called Primary Cell Group, PCG) whereas the additional radio link provided by Small Cell acts as a local capacity booster (i.e. Secondary Cell Group, SCG). This feature facilitates Control Plane/User Plane (CP/UP) split, in order to reduce the signaling overhead, reduce the number of handovers, and to improve mobile users' experience. $\mathrm{CP} / \mathrm{UP}$ split enables to switch the UP links among the available $\mathrm{SCs}$, whereas the user's context $(\mathrm{CP})$ is maintained by the overlay macro-cell. In contrary to CA, DC scheme uses concept of the Split Bearer, where instead of aggregating MAC layer transport blocks, the PDCP Packet Data Units (PDUs) are combined, thus omitting the requirement for low latency and allowing non-ideal backhaul for SC connectivity.

Rel-13 extends the spectrum aggregation mechanisms towards the higher number of the aggregated bands and towards the use of unlicensed spectrum for mobile networking. Massive CA enables to use up to 32CCs and thus theoretically provides up to $640 \mathrm{MHz}$ of aggregated bandwidth for a single device, while still fulfilling backwards compatibility with LTE Rel-8 channel bandwidths. 
Figure 3. Two variants for LTE RAN network elements reconfiguration under LSA scheme [14, 15]

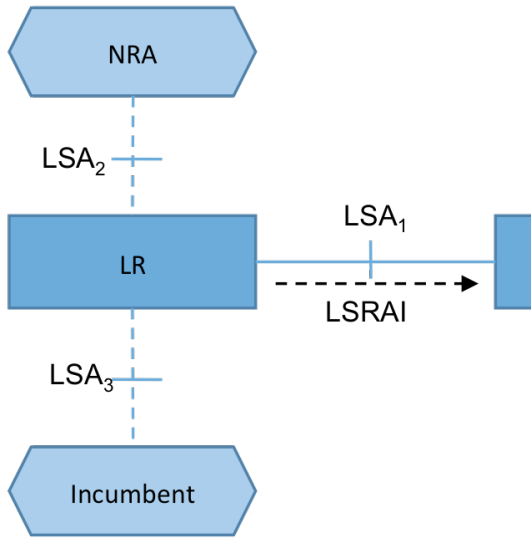

Referring to the CA band combinations defined in 3GPP, it can be observed, that most of the CA band combinations are $\mathrm{DL}$ focused, i.e. more $\mathrm{CC}$ are allocated for the $\mathrm{DL}$ traffic, than for the UL, e.g. 3 DL CC and 1 UL CC. This can be motivated by DL-heavy applications putting requirements on more network capacity for DL. Moreover, a typical UE can support only 1 or 2 UL CCs. In case of TDD bands, such CA arrangement could lead to problems for channel reciprocity exploitation. This is due to UE limitations in the number of supported UL CCs, i.e. smaller number of UL carriers can be aggregated, than DL carriers. In consequence some of TDD carriers with DL transmission, could not have UL transmission with SRS reference signals for the channel estimation, and therefore the channel reciprocity could not be exploited. This limitation could be highly visible, if we consider the $32 \mathrm{CC}$-version of CA, where the large portion of CC's are provided in TDD bands.

Thus, to overcome the above TDD issue, a work item called SRS Carrier base Switching for LTE [11] has been agreed for Rel-14. It aims to introduce fast carrier switching to and among TDD UL component carriers to allow the SRS transmission on those TDD carriers. Specifically, CC used for the SRS transmission corresponds to the $\mathrm{CC}$ available for PDSCH, and the UE has fewer CCs available for PUSCH. Furthermore, this work will cover also the SRS signalling reduction techniques, similar to Lean Carrier or NCT concepts.

In terms of unlicensed spectrum, LAA and LTE-WiFi Aggregation (LWA $)^{\dagger \dagger \dagger}$ are provided as features to utilize it in mobile networks. LAA aggregates the licensed LTE carrier (serving as a mobility and signaling anchor - PCell) with SCell using the new LTE frame format over the unlicensed $5 \mathrm{GHz}$ ISM band ${ }^{+*}$. Similarly, in the case of LWA scheme, the Carrier Wi-Fi is serving as a capacity

\footnotetext{
${ }^{\dagger \dagger}$ All spectrum access and spectrum aggregation schemes have deployment related limitations, which are not discussed in this paper, e.g. LWA and LAA are applicable mostly for Small Cells scenarios. \$* Other LTE-based access schemes using ISM spectrum: LTE-U downlink-only radio access with Carrier Sensing Adaptive Transmission (CSAT) for fairness assurance; MuLTEfire - LTE-based technology without licensed PCell anchor proposed by Nokia and Qualcomm.
}

booster counterpart using the radio level integration for uniform user experience provisioning over the WiFi. In LWA, UE is configured by the eNB to utilize radio resources of LTE and WLAN.

As LWA standardized within Rel-13 considers DL-only operation, an enhanced LWA (eLWA) is proposed within Rel-14 [12] to overcome this limitation and builds on the already existing LWA framework. The new features envisioned for this enhancement include:

- Addition of UL transmission via WLAN (including bearer split),

- $\quad$ Support for e.g. $60 \mathrm{GHz}$ and for PDCP optimizations for increased data rates,

- SON-related features for WLANs under eNB coverage (e.g. Automatic Neighbor Relation).

Another LTE-WLAN interworking mode is called RAN Controlled LTE WLAN Interworking (RCLWI), defining LTE-controlled bi-directional traffic steering between LTE and WLAN. In RCLWI, LTE may send steering commands to the UE in order to perform traffic offloading to WLAN. LWA and RCLWI interworking schemes are supported in collocated, as well as in non-collocated scenarios (i.e. ideal backhaul, or non-ideal backhaul between eNB and WLAN Access Point (AP), respectively).

\subsection{Spectrum licensing schemes}

Licensed spectrum allocation per Mobile Network Operator (MNO) is the basic principle for the mobile networks operation, requiring acquisition of the spectrum license. However, an unlicensed spectrum usage was considered already in 3GPP Rel-8, where the Access Network Discovery and Selection Function (ANDSF) was introduced for the traffic offload to e.g. Carrier Wi-Fi AP's. This technique was not very popular, but the traffic offloading evolution continued in subsequent $3 \mathrm{GPP}$ releases.

As already mentioned in the previous section, the $3 \mathrm{GPP}$ has introduced LAA, an unlicensed spectrum access scheme, which became a hot topic among the 3GPP, WFA and IEEE. ISM spectrum usage was considered under the LWA or RCLWI schemes. To enhance the integration of unlicensed 
spectrum into mobile networks, the Unlicensed Spectrum Offloading System (USOS) work was also agreed for Rel-14. It aims to define service requirements to enable an MNO to understand unlicensed spectrum usage and to allow for its accounting for network planning and charging purposes.

Furthermore, the new spectrum sharing scheme for 2.3-2.4 GHz band was introduced, based on the ETSI Reconfigurable Radio Systems (RRS) work [13], called License Shared Access (LSA, or Authorized Shared Access, ASA). LSA allows spectrum owners (i.e. Incumbents) to share their radio resources with other market players (i.e. LSA Licensees), enabling QoS support within a shared band. QoS support is achieved by the use of protection measures such as geographical exclusion, or restriction zones, within which the Incumbent's receivers will not be subject to interference caused by LSA Licensees. 3GPP studied LSA access in Rel-13 [14], looking into architectural aspects of ETSI's concept for global solution provisioning, covering required information flows for static and semistatic spectrum sharing scenarios. Two alternatives for OAM based spectrum usage reconfigurations are available within the LSA architecture model to handle the LSA Spectrum Resource Availability Information (LSRAI) exchange over the LSA 1 interface (see Figure 3), namely direct provisioning of LSRAI or providing network reconfiguration. LSA Repository (LR) entity stores information describing the Incumbent's usage and protection requirements. LR allows National Regulatory Authority (NRA) to monitor the LSA operation. LSA Controller (LC) is located within the licensee's domain, allowing the licensee to obtain the LSA spectrum availability information from the LR. LC interacts with the LSA Licensee's mobile network to support mapping of the spectrum availability information into appropriate radio nodes configurations.

\subsection{Duplexing schemes}

Duplexing schemes for the LTE system correspond to either FDD or TDD, with pre-defined duplex-specific spectrum bands [5]. The initial Rel-8 TDD frame configurations included multiple settings for different UL and DL traffic ratios, ranging from UL heavy $2: 2: 6^{\S \S}$ up to DL heavy $8: 1: 1$, within $10 \mathrm{~ms}$ radio frame, to be configured in a semistatic manner. However, TD-LTE frame setup modification requires careful inter-site coordination to avoid major interference problems, e.g. in case of inter-site configuration, where UL and DL transmission is present in the same subframe from the neighboring access nodes.

Based on the TDD frame configurations described above, their dynamic adaptation for a HetNet scenario was introduced in Rel-12 with the introduction of the enhanced Interference Management and Traffic Adaptation (eIMTA) feature. eIMTA enables on-the-fly changes of the TDD configuration based on instantaneous DL:UL traffic demand ratio in the particular cell. This feature was mainly foreseen

$\S \S \S$ DL:Sp:UL; Sp - Special subframe for Small Cell deployments in $3.5 \mathrm{GHz}$ band. In such scenario, DL-UL interference should not be an issue, since a SC with low transmit power, and operating on high frequency band is considered to be well isolated from the potential neighboring interferers (unless, dense deployment of SCs is considered), and it is not expected to deploy macro cells on such high frequency bands ${ }^{* * * *}$.

Furthermore, TDD frame configurations were discussed within Rel-13 to be further extended with additional DL-heavy and DL-only frame formats to support 9:1:0 and 10:0:0 options [16], but this study was left for standardization in future releases.

With the introduction of CA, TDD or FDD spectrum aggregation was possible. In case of FDD bands aggregation, it was possible to have a different number of the DL and UL CCs (e.g. refer to the SDL concept provided in section 3.3 ); in the case of TDD spectrum aggregation, number of carriers (and their bandwidths) obviously has to be the same for DL and UL due to the nature of TDD. Further evolution of CA configurations came with the aggregation of FDD and TDD component carriers, enabling greater flexibility in the spectrum arrangements for the operators having both, FDD and TDD spectrum licenses. In such a scenario, PCell could be either FDD, or TDD (with the limit for the cross-carrier scheduling originating from the TDD PCell). One particular use case considers FDD based PCell, with the RRH based SCell using TDD.

Flexible duplex feature for the FDD E-UTRA bands was also proposed during Rel-13 discussions [17]. It aimed to provide more efficient handling of the asymmetric traffic load between DL and UL, going beyond static resource allocation for both link directions, by permitting DL transmission to originate from the underutilized FDD UL frequency bands. Study on the flexible duplex was not concluded yet, but based on negative feedback received from regulatory bodies, it seems that the option of flexible duplex for FDD bands might not reach the normative phase of standardization.

\subsection{Spectrum sharing schemes}

According to the basic definition of spectrum sharing ${ }^{\dagger \dagger \dagger}$, some of the techniques described in the previous chapters could be considered as belonging to the spectrum sharing mechanisms, e.g. LAA as a spectrum access method, or LSA as a spectrum licensing method.

This section focuses on the recent developments in the area of shared spectrum access improvements, in the form of Rel-13 RAN Sharing Enhancements (RSE) work [18]. RSE treats on the RAN sharing, however it is closely related to spectrum sharing. It improves the legacy inter-operator sharing schemes, where previously there was no knowledge

\footnotetext{
However, 3.5GHz based rooftop macro sites were considered in Japan, based on the inter-site coordination for interference management purposes.

tit Spectrum sharing: simultaneous usage of the particular frequency band by the number of independent systems, or users.
} 
on how much capacity the other participating operator is using. RSE feature is addressing the following aspects:

- Allocation of the shared RAN resources based on the proportion of the assigned resource usage for each participating operator,

- Ability to monitor the usage of shared resources,

- Allowing on-demand capacity negotiations,

- Load balancing while respecting the agreed shares of resources,

- Selective OAM access for the participating operators.

RSE is under the development for each of the following radio technologies: GSM, UTMS [19] and LTE. In the case of GSM, it is becoming an interesting improvement for some markets, where it is already expected, that there will be a single shared GSM network (to support legacy devices) for all competing operators. At the same time, each MNO will own its individual $4 \mathrm{G}$ network using extra $2 \mathrm{G}$ spectrum resources by the means of dynamic refarming.

RSE functionality is expected to be particularly important in case of HetNets, including dense deployment of SCs for hotspots. In the authors' opinion, SC sharing will become an enabler for fruitful deployments of future networks in mature markets, where shortage of the access nodes' sites in dense environments will become a bottleneck.

\subsection{Spectrum refarming and Multi-RAT coordination}

Along the mobile networks evolution from $1 \mathrm{G}$ analogue systems up to today's LTE-A Pro cellular networks, underlying spectrum regulations and standardization have been evolving to provide sufficient amount of market-, and RAT-specific frequency bands for the mobile operators. With the new radio technologies becoming more spectrally efficient than the previous RAT generations and with an ever-increasing demand for the more licensed bands, the spectrum refarming comes as a natural solution to enable releasing of the legacy spectrum bands. Static approach to the spectrum refarming, where reuse of the $2 \mathrm{G}$ spectrum for the LTE services is allowed after $2 \mathrm{G}$ license expiration, is not too attractive, as market-specific spectrum licenses might have been granted for long periods (e.g. 20 years). Moreover, the static spectrum refarming has another drawback, i.e. the requirement of legacy RAT's devices lead-out. Thus, the evolution in spectrum refarming domain already includes dynamic methods, and ultimately shifts towards Cognitive Radio-based mechanisms.

So far, one promising method for dynamic spectrum refarming is being standardized under Multi-RAT Joint Coordination framework [20, 21]. This concept utilizes Dynamic Spectrum Access (DSA) scheme + , where the collocated LTE and GSM systems use the dedicated bandwidth parts, whose sizes depends on the actual traffic

$\$ 4$ Also studied in FP7 SEMAFOUR project [24]. demand in each of those radio networks. This method is based on the temporal traffic statistics, e.g. when GSM load on Traffic Channel is low, LTE is allowed to use shared GSM/LTE part of the spectrum.

As an evolution of the studies on coordinated resources utilization in MRAT Joint Coordination, study on HSPA and LTE Joint Operation [22] has been approved for Rel-14. Considering the support of voice services in Packet Switched (PS) based LTE, and its reliance on the Circuit Switched (CS) Fallback (CSFB) to the UMTS network being a common mechanism, it is expected that such setup of parallel CS- and LTE PS-based voice services will take a while. This example cooperation mechanism poses a significant restriction from the user's point of view, who is allowed to use radio resources from a single RAT (and single channel quality), at a time. In such case, a user can camp and access one RAT and be scheduled to perform inter-RAT mobility procedures for load balancing or overload prevention, etc. Therefore, it was proposed to study solution that allows tighter inter-RAT integration, where voice services are provided via wide-area UMTS network, while high data rates can be provided via LTE. In other words, CS is provided via UMTS and PS via LTE, in parallel. This approach is an alternative way to the spectrum refarming, given the fact that the data traffic in some HSPA networks is still increasing.

\section{Future evolution directions}

To provide a qualitative comparison of the Spectrum Toolbox' elements, an analysis of their potentials and challenges is presented in Table 4. Considering the above solutions and the emerging standardization of $5 \mathrm{G}$, the presented Spectrum Toolbox is expected to be further enhanced with the new technology elements. Thus, it will become even more complex, but it will also provide more flexibility in the spectrum allocation. Because of that, Spectrum Toolbox will have to be more adaptive and automated, evolving towards cognitive mechanisms, equipped with self-learning and self-optimization solutions.

Some of the 3GPP studies and work items considered for Rel-14 discussion, are indicating future evolution directions, as briefly summarized below:

- Enhanced LAA (eLAA) extends the existing LAA scheme with UL consideration to enable full DC-like capabilities for unlicensed spectrum [23].

- SDL carriers to be defined for 2570-2620MHz band for TDD or for unpaired DL within SDL framework [9].

- Lean Carrier concept (after being initially rejected in Rel-12) is gaining attention again [25]. It is supposed to inherit from LAA radio frame, where most of the legacy PHY-layer information (including cell specific reference signals) is sent only when needed. By doing so, in case of low/medium network load conditions, the interference level caused by transmission of signaling is decreased, and the eNB power amplifiers can be switched to deep-sleep-mode in order to save energy. 
Furthermore, to enable easy integration with the existing system, it is proposed to provide the lean carrier with the possibility to be toggled to the legacy LTE frame when needed e.g., to serve as a legacy SCell.

- Multi-connectivity [26] is expected to enhance DC, by providing multiple radio links for a UE in two following options: first option considers a configuration of multiple radio links per UE, where only limited, selected set of radio links is active at any given moment. Alternatively, all of the configured multiple radio links could be active.

- To improve the spectrum utilization within existing system, two study items are proposed for Rel-14, namely: enabling light connection via signaling reduction [27] and study on latency reduction [28] for LTE. Both of them bring ideas of addressing $5 \mathrm{G}$ use cases already within LTE-Advanced Pro. The former focuses on the user-centric mobility approach to decrease handover-related signaling and a new intermediate RRC state for keeping UE context alive during short active/inactive transitions. The latter aims at decreasing UP latency via PHY layer optimizations. Among them, shortening TTI down to a single EUTRA OFDMA symbol and more resource efficient UL scheduling timing are the most interesting ones.

More advanced and novel areas of the "Towards 5G" RAN aspects include: adoption of the high frequency bands, support for full duplex, and definition of the Unified Air Interface (UAI):

- New frequency bands in the range of mmWave bands have been considered for local capacity boosting and to serve as dynamic backhaul/fronthaul solutions, where the standardization starts by studying the appropriate channel models (mentioned in section 2.2).

- Full duplex provides the possibility to improve spectral efficiency by utilizing a single, un-paired band for simultaneous transmission and reception (i.e. without splitting the time slots onto DL and UL) possible through the use of advanced receivers.

- UAI should handle multiple different traffic types and incorporate their requirements into a single radio frame design utilizing different frame parts (e.g. in the frequency domain) with different waveform parameters and access schemes. For example, small data packets transmitted by MTC devices can be sent in a contention-based manner, whereas MBB data is sent in synchronized scheduled manner [29].

All the above provides a Spectrum Toolbox that includes a wide set of bands ranging from $450 \mathrm{MHz}$ up to $100 \mathrm{GHz}$ with the licensed and unlicensed spectrum, covering different licensing options, as well as different access schemes, BW aggregation mechanisms, duplexing schemes and RATs. On top of that, $5 \mathrm{G}$ requirements target tight integration of all these elements to unify the operation of the next generation mobile systems and to provide the possibility to adapt to different use cases and scenarios that will further complicate the overall mobile systems landscape.

3 GPP has already initiated work on " $5 \mathrm{G}$ " Air Interface under the umbrella of Rel-14 within study item on New Radio Access Technology [30] that to large extent will cover the presented Spectrum Toolbox. To be more specific, according to the study item description, the NR radio is expected to:

- Cover frequencies to up to $100 \mathrm{GHz}$,

- Act as a framework jointly addressing all use cases including: enhanced Mobile Broadband, massive MTC and ultra-reliable communications,

- Should be forward compatible to enable easy introduction of new features,

- Should be tightly integrated with LTE and make interworking with Wi-Fi possible,

- Should enable operation in licensed, licensed-shared and license-assisted bands.

\section{Summary and conclusions}

The aim of this article is to present the set of the already available and currently studied spectrum access techniques, considered as an enabler for the $5 \mathrm{G}$ pre-standardization discussions within 3GPP Rel-14. Based on the performed analysis and as indicated in [31], the authors are convinced that the ongoing 5G-related debates should already address all the possible spectrum resources, while considering various licensing schemes. A unified approach to the complex problem of RRM is an enabler for the spectrumefficient future mobile networks. This is due to the fact, that the unified approach to RRM is still considered to be a missing piece in the current multi-RAT mobile networks. In order to make an efficient usage of the new spectrum licensing schemes possible (like LSA), considering scenarios which are evolving towards Ultra Dense Networks (UDN), the radio resources coordination shall be addressed on multiple levels, namely inter-MNO, inter-RAT, inter-site, inter-layer, inter-band dimensions. To achieve this, it is already obvious that the high level flexibility in the RF domain will be required. Additionally, a high level of "programmability" of the baseband units will be needed, relying on Software Defined Radio (SDR), leading to the dynamic spectrum access. On top of that, the overall design of future networks should natively incorporate SON engines, to manage the network towards a unified user experience provided across multiple converged radio access technologies. By looking onto the latest $3 \mathrm{GPP}$ study on the New Radio targeting the specification of $5 \mathrm{G}$, it can be easily deducted that it covers the Spectrum Toolbox described in this article. Therefore, the authors believe that the material presented above will help the readers to navigate through the already complicated spectrum access landscape. 
Table 4. Advantages and challenges of the Spectrum Toolbox features

\begin{tabular}{|c|c|c|}
\hline Feature & Advantages and opportunities & Disadvantages and challenges \\
\hline $\begin{array}{c}\text { Carrier } \\
\text { Aggregation }\end{array}$ & $\begin{array}{ll}\text { - } & \text { Improves user throughput and cell } \\
\text { - } & \text { capacity } \\
\text { Possibility to aggregate different } \\
\text { - } \\
\text { - } \quad \text { MACtension beyond single carrier allocation } \\
\text { - MAC management }\end{array}$ & $\begin{array}{ll}\text { - } & \text { Not possible to aggregate spectrum in } \\
\text { - } & \text { non-ideal backhaul RRH deployments } \\
\text { - } & \text { Multiple market-specific CA band combinations }\end{array}$ \\
\hline $\begin{array}{l}\text { Massive Carrier } \\
\text { Aggregation }\end{array}$ & $\begin{array}{l}\text { - } \quad \text { Extension up to } 32 \mathrm{CCs} \\
\text { Enables to acquire multitude of bands } \\
\text { and BWs to increase capacity and mix } \\
\text { licensed with unlicensed bands }\end{array}$ & $\begin{array}{ll}\text { - } & \text { Complex management } \\
\text { - } & \text { Complexity of RF chains } \\
\text { - } & \text { UE support to be limiting factor }\end{array}$ \\
\hline $\begin{array}{l}\text { Supplemental } \\
\text { Downlink }\end{array}$ & 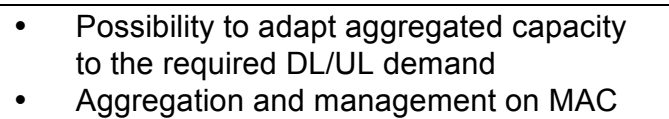 & $\begin{array}{l}\text { - Feature limited by the available SDL-specific } \\
\text { bands } \\
\text { - } \quad \text { CA-based operation only }\end{array}$ \\
\hline $\begin{array}{c}\text { Dual } \\
\text { Connectivity }\end{array}$ & $\begin{array}{ll}\text { - } & \text { Adds spectrum aggregation opportunity } \\
\text { - } & \text { Enar non-ideal backhaul inter-site } \\
\text { - } & \text { Possible to combine with CA } \\
\text { - } & \text { Enables extension to aggregate } \\
& \text { multi-RAT aggregation on PDCP level }\end{array}$ & $\begin{array}{l}\text { - Not possible to allocate resources on MAC } \\
\text { level } \\
\text { May have problems at anchor cell boundary } \\
\text { due to both Macro and SC change } \\
\text { - Requires an additional scheduler }\end{array}$ \\
\hline $\begin{array}{c}\text { Multi } \\
\text { Connectivity }\end{array}$ & $\begin{array}{l}\text { - } \quad \text { Extension of DC for UDN } \\
\text { Enabler for mmW bands support due to } \\
\text { blocking problems (mobility anchors) } \\
\text { Reduces the impact of throughput } \\
\text { decrease at macro cell boundary by the } \\
\text { use of "make-before-break" concept }\end{array}$ & - $\quad$ Complex management \\
\hline $\begin{array}{l}\text { LTE-WiFi } \\
\text { Aggregation }\end{array}$ & $\begin{array}{ll} & \text { Enables to aggregate multiple-RATs } \\
\text { - } & \text { Use of free unlicensed spectrum } \\
\text { - } & \text { Enables use of widely available } \\
& \text { technology } \\
\text { - } & \text { Most terminals support WiFi already } \\
\end{array}$ & $\begin{array}{ll}- & \text { Requires carrier WiFi access nodes } \\
\text { deployment } \\
\text { - } & \text { Requires additional scheduler at PDCP level } \\
\text { - } & \text { Doesn't support interference management in } \\
& \text { ISM bands } \\
\end{array}$ \\
\hline $\begin{array}{l}\text { License } \\
\text { Assisted } \\
\text { Access }\end{array}$ & $\begin{array}{ll} & \text { Use of free unlicensed spectrum } \\
\text { - } & \text { Improves Return Over Investment (ROI) } \\
\text { - } & \text { Most terminals support WiFi bands } \\
& \text { already }\end{array}$ & $\begin{array}{ll}\text { - } & \text { Doesn't support interference management in } \\
\text { ISM bands } \\
\text { - } \quad \text { Fair spectrum sharing with WiFi required } \\
\text { - } \quad \text { Lower spectral efficiency than in licensed } \\
\text { bands }\end{array}$ \\
\hline $\begin{array}{l}\text { Dynamic } \\
\text { Spectrum } \\
\text { Access }\end{array}$ & $\begin{array}{ll} & \text { Enable easy on-demand spectrum } \\
\text { - } & \text { Pefarming } \\
\text { Possibility to adjust capacity to actual } \\
\text { demand in a dynamical manner } \\
\text { - Limits guardband gaps that are imposed } \\
\text { by static refarming }\end{array}$ & $\begin{array}{l}\text { - Gains limited by the amount of the legacy } \\
\text { spectrum to be refarmed (i.e. } 2 \mathrm{G} \text { and/or } 3 \mathrm{G} \text { ) }\end{array}$ \\
\hline FDD & $\begin{array}{l}\text { Easier resources management due to } \\
\text { decoupled DL and UL (separate } \\
\text { schedulers) } \\
\text { Interference management less complex } \\
\text { than TDD (at UE side) }\end{array}$ & $\begin{array}{l}\text { In case of non-symmetrical DL/UL traffic, } \\
\text { non-optimal spectrum resources allocation }\end{array}$ \\
\hline TDD & $\begin{array}{l}\text { - Improves spectral efficiency in case of } \\
\text { non-symmetrical traffic between DL/UL } \\
\text { Allows channel reciprocity for channel } \\
\text { estimation }\end{array}$ & $\begin{array}{l}\text { - Not possible to dynamically adapt DL/UL share } \\
\text { to traffic demand } \\
\text { Requires inter-site coordination for } \\
\text { interference avoidance }\end{array}$ \\
\hline eIMTA & $\begin{array}{l}\text { Enables dynamic on-demand UL/DL } \\
\text { adaptation of resources allocation } \\
\text { Boosts TDD advantages }\end{array}$ & $\begin{array}{ll}\text { - } & \text { Requires isolation between cells for DL/UL } \\
\text { interference avoidance } \\
\text { - } & \text { Requires proper site coordination in case when } \\
\text { SCs are not isolated } \\
\text { - Application to high frequency bands (due to cell } \\
\text { isolation requirement) }\end{array}$ \\
\hline
\end{tabular}




\begin{tabular}{|c|c|c|}
\hline Feature & Advantages and opportunities & Disadvantages and challenges \\
\hline Full Duplex & $\begin{array}{ll}\text { - } & \text { High spectral efficiency } \\
\text { - } & \text { Independency of DL/UL traffic share }\end{array}$ & $\begin{array}{ll}\text { - } & \text { Implementation and receiver complexity } \\
\text { - } & \text { Interference management complexity } \\
\text { - } & \text { Non-backward compatible terminals } \\
\end{array}$ \\
\hline $\begin{array}{c}\text { Flexible } \\
\text { bandwidth }\end{array}$ & $\begin{array}{ll}- & \text { Improves refarming of legacy bands } \\
\text { - } & \text { Improves granularity of individual } \\
\text { spectrum blocks for aggregation }\end{array}$ & $\begin{array}{ll}-\quad & \text { No gains for legacy devices } \\
\text { - } & \text { Additional mechanisms needed to support } \\
& \text { legacy UEs }\end{array}$ \\
\hline $\begin{array}{l}\text { Sub-6GHz } \\
\text { spectrum }\end{array}$ & $\begin{array}{ll} & \text { Wide area access (lower bands) } \\
\text { - } & \text { NLOS operation possible } \\
\text { - } & \text { Good for high mobility (lower bands) }\end{array}$ & $\begin{array}{ll}\text { - } & \text { Limited availability (most bands highly } \\
\text { occupied) } & \\
\text { - } & \text { Fragmented spectrum chunks (lack of large } \\
\text { contiguous spectrum blocks) } \\
\text { - Limited use of Massive MIMO due to antenna } \\
\text { form factors (both BS and UE side) }\end{array}$ \\
\hline $\begin{array}{l}\text { mmWave } \\
\text { spectrum }\end{array}$ & $\begin{array}{l}\text { - Availability of large contiguous spectrum } \\
\text { chunks } \\
\text { Enables use of Massive MIMO due to } \\
\text { small antenna elements sizes }\end{array}$ & $\begin{array}{ll}\text { - } & \text { Short range only (depending on scenario) } \\
\text { - } & \text { Severe blocking effects } \\
\text { - } & \text { New channel models required } \\
\text { - } & \text { LOS only operation } \\
\end{array}$ \\
\hline $\begin{array}{l}\text { Unified Air } \\
\text { Interface }\end{array}$ & $\begin{array}{ll}\text { - } & \text { Flexible use of frequency bands } \\
\text { - } & \text { PHY adaptation to traffic demand } \\
& \text { Possibility to incorporate different } \\
& \text { waveforms parameters } \\
\end{array}$ & $\begin{array}{ll}\text { - } & \text { High complexity } \\
\text { - } & \text { Limited gains for legacy devices }\end{array}$ \\
\hline $\begin{array}{l}\text { RAN Sharing } \\
\text { Enhancements }\end{array}$ & $\begin{array}{l}\text { Enables on-demand capacity negotiation } \\
\text { between operators } \\
\text { - Semi-dynamic approach for adapting } \\
\text { spectrum portion share to traffic demands } \\
\text { between operators } \\
\text { - } \quad \text { Shared resources usage monitoring } \\
\end{array}$ & $\begin{array}{ll} & \text { Sharing arrangement parties are licensees } \\
\text { - } & \text { Not fully dynamic mechanism }\end{array}$ \\
\hline $\begin{array}{c}\text { Licensed } \\
\text { Shared Access }\end{array}$ & 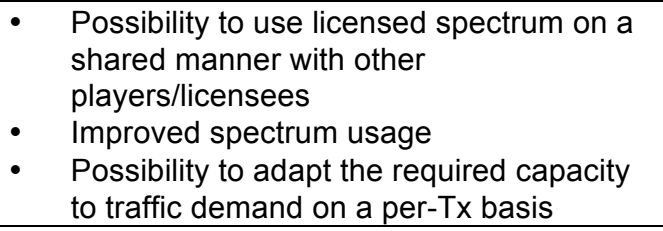 & $\begin{array}{l}\text { - } \quad \text { Limited to certain bands } \\
\text { Semi-dynamic behaviour, not possible to adapt } \\
\text { spectrum availability in a highly dynamic } \\
\text { manner in case of fast changes of traffic within } \\
\text { Small Cell }\end{array}$ \\
\hline $\begin{array}{c}\text { Small Cell } \\
\text { sharing (SCaaS) }\end{array}$ & $\begin{array}{ll}\text { - } & \text { Decouples infrastructure provider (e.g. } \\
\text { - } & \text { Infrast estate owner) from MNO } \\
\text { - } & \text { Dynamic use of spectrum adapting to } \\
\text { traffic demand } \\
\text { - } \\
\text { Multi-RAT compatible }\end{array}$ & $\begin{array}{ll}\text { - } & \text { Lack of control of the infrastructure } \\
\text { - } & \text { Inter-MNO coordination for congestion situation }\end{array}$ \\
\hline
\end{tabular}

\section{References}

[1] 3GPP, "Report of 3GPP RAN workshop on LicensedAssisted Access (LAA)", (2015)

[2] D. Flore, "RAN workshop on 5G: Chairman Summary", 3GPP RAN, RWS-150073, (2015)

[3] 3GPP, RP-151606, "Study on channel model for frequency spectrum above $6 \mathrm{GHz}$ ", (2015)

[4] Ericsson, "ITU WRC-15 summary", (2015)

[5] 3GPP, TS36.104, "Base Station (BS) radio transmission and reception", v13.1.0, (2015)

[6] 3GPP, RP-160681, "LTE bandwidth flexibility enhancements", (2016)

[7] 3GPP, TR37.814, "L-band for Supplemental Downlink", (2014)

[8] 3GPP, RP-152042, “TR 36.895 v1.0.0 European 700 Supplemental Downlink band $(738-758 \mathrm{MHz})$ in EUTRA", (2015)
[9] 3GPP, RP-152004, "LTE FDD 2.6 GHz SDL band (2570$2620 \mathrm{MHz})$ ", (2015)

[10] 3GPP, RP-151671, "LTE Advanced inter-band CA Rel-14 for 5DL/1UL", (2015)

[11] 3GPP, RP-160676, "SRS Carrier based Switching for LTE", (2016)

[12] 3GPP, RP-160600, "New Work Item on enhanced LWA", (2016)

[13] ETSI 103 154, "System requirements for operation of Mobile Broadband Systems in the $2300 \mathrm{MHz}-2400 \mathrm{MHz}$ band under LSA", v1.1.1, (2014)

[14] 3GPP, TR32.855, "Study on OAM support for LSA", v0.4.0, (2015)

[15] 3GPP, SP-150056, "Study on OAM support for LSA", (2015)

[16] 3GPP, RP-142248, "Study on possible additional configuration for LTE TDD", (2015)

[17] 3GPP, RP-150470, "Study on regulatory aspects for flexible duplex for E-UTRAN", (2015) 
[18] 3GPP, RP-130330, "RAN Sharing Enhancements", (2015)

[19] 3GPP, SP-140637, "GERAN UTRAN Sharing Enhancements", (2014)

[20] 3GPP, RP-150417, "Study on Multi-RAT Joint Coordination", (2015)

[21] 3GPP, TR37.870, "Study on Multi-RAT joint coordination", (2015)

[22] 3GPP, RP-160571, "Study on HSPA and LTE Joint Operation", (2016)

[23] 3GPP, RP-151978, "New Work Item on enhanced LAA for LTE”, (2015)

[24] 3GPP, RP-151889, "LTE bandwidth flexibility enhancements", (2015)

[25] 3GPP, RP-151982, "New Work Item on Lean Carrier for LTE”, (2015)

[26] 3GPP, RP-151791, "Enhancements for densely deployed small cells in LTE”, (2015)

[27] 3GPP, RP-160540, "Signalling reduction to enable light connection for LTE", (2016)

[28] 3GPP, RP-150465, "Study on Latency reduction techniques for LTE", (2016)

[29] G. Wunder, et. al, "5GNOW: Non-Orthogonal, Asynchronous Waveforms for Future Mobile Applications", Communications Magazine, (2014)

[30] 3GPP, RP-160671, "Study on NR New Radio Access Technology", (2016)

[31] M. Szydelko, M. Dryjanski, "Spectrum Toolbox Survey: Evolution Towards 5G", Proc. The Eleventh EAI International Conference on Cognitive Radio Oriented Wireless Networks (CROWNCOM), Grenoble, France, (2016) 